\title{
Qualitätssicherung in der Langzeitpflege durch Wahlent- scheidungen der Betroffenen? Eine ökonomische Perspektive
}

\author{
Stefan Greß \\ (c) Der/die Autor(en) 2018 \\ K. Jacobs et al. (Hrsg.), Pflege-Report 2018 \\ https://doi.org/10.1007/978-3-662-56822-4_13
}

\section{Zusammenfassung}

Der Gesetzgeber hat in der sozialen Pflegeversicherung Wahlentscheidungen der Betroffenen auf dem Leistungsmarkt einen hohen Stellenwert eingeräumt. Pflegebedürftige bzw. deren Angehörige können ihre Wahlentscheidungen derzeit jedoch nur auf der Grundlage unvollständiger Informationen treffen. Ohne aussagefähige Informationen über wichtige Struktur-, Prozess- und Ergebnisparameter läuft eine Qualitätssicherung über individuelle Wahlentscheidungen ins Leere. Die Gründe für die eingeschränkte Funktionsfähigkeit von Wahlentscheidungen auf dem Leistungsmarkt blieben jedoch auch bei einer nachhaltigen Verbesserung der Informationsgrundlage weitgehend bestehen.

The legislator has accorded a high priority in the long term care insurance system to the decisions of persons concerned, but patients and their relatives are currently only able to make their choice on the benefits market on the basis of incomplete information. Without meaningful information about important structure, process and outcome parameters, quality assurance is rendered meaningless by individual decisions. However, even if the information basis were improved in a sustainable way, the reasons for the limited effectiveness of individual decisions on the benefits market would largely remain unchanged.

\subsection{Einleitung}

Aus ökonomischer Sicht dienen die Steuerungsmechanismen Markt und Wettbewerb dazu, dass Verbraucher durch ihre Wahlentscheidungen Unzufriedenheit signalisieren können. Qualitativ unzureichende Leistungsangebote bzw. Leistungsanbieter würden demnach auf einem Markt nicht nachgefragt werden und müssten ihre Qualität verbessern oder aus dem Markt ausscheiden. Die Funktionsfähigkeit dieses Mechanismus ist jedoch nicht voraussetzungslos. Insbesondere muss auf der
Nachfrageseite der »Homo oeconomicus« über hinreichende Informationen und Handlungsoptionen verfügen und diese in geeigneter Art und Weise verarbeiten können, um unterschiedliche Leistungsangebote miteinander vergleichen zu können.

Dieser Beitrag geht der Frage nach, inwieweit Wahlentscheidungen auf der Nachfrageseite dazu führen können, die Qualität in der Langzeitpflege zu sichern. ${ }^{1}$ Dazu stellt er die Bedeutung von Wahl-

1 In diesem Beitrag werden die Ausführungen des Autors aus einem früheren Aufsatz ergänzt und aktualisiert (Greß 2017). 
entscheidungen der Verbraucher einschließlich der Voraussetzungen für deren Funktionsfähigkeit auf der Nachfrageseite dar. Im zweiten Schritt wird dieses Konzept auf den Markt für Leistungen der Langzeitpflege angewandt. Im Anschluss wird analysiert, inwieweit die Wahlentscheidungen hinsichtlich dieses Leistungsangebots dem Idealbild der Steuerung durch Markt und Wettbewerb entsprechen. Aus den Grundproblemen des vorherrschenden Steuerungsmodells in der gesetzlichen Pflegeversicherung werden Überlegungen abgeleitet, wie die Wahlentscheidungen möglichst optimiert werden können und wie wirksam ergänzende Steuerungsmechanismen sind. Die abschließende Diskussion fasst die zentralen Erkenntnisse dieses Beitrags zusammen.

\subsection{Voraussetzungen für die Wirk- samkeit von Wahlentscheidungen}

Aus ökonomischer Sicht ist der Steuerungsmechanismus Markt der zentrale Allokationsmechanismus, um knappe Ressourcen alternativen Verwendungsmöglichkeiten möglichst effizient zuteilen zu können. Im Zentrum dieses Mechanismus steht der einzelne Verbraucher, der durch seine Wahlentscheidungen auf der Grundlage seiner Präferenzen den individuellen Nutzen optimiert. Nur der Verbraucher ist danach in der Lage, individuelle Abwägungen zu Kosten und Nutzen alternativer Verwendungsmöglichkeiten $\mathrm{zu}$ treffen. Insofern ist es zentral für die Wirksamkeit von Wahlentscheidungen, dass der Verbraucher seine Entscheidungen ohne Eingriffe von Dritten auf der Grundlage seiner individuellen Vorlieben treffen kann. Mit anderen Worten: Die Funktionsfähigkeit des Marktes hängt davon ab, dass eine möglichst weitreichende Konsumentensouveränität bei der Wahlentscheidung auf der Grundlage individueller Präferenzen bzw. Vorlieben herrscht. Eingriffe von Dritten - insbesondere des Staates sind in dieser Logik begründungspflichtig, weil sie individuelle Abwägungen zumindest in Teilen außer Kraft setzen (Rice und Unruh 2016).

Für die Funktionsfähigkeit des Marktes ist allerdings nicht nur entscheidend, dass Verbraucher in ihren Wahlentscheidungen nicht von Dritten beeinflusst werden. Es müssen vielmehr weitere Voraus- setzungen auf der Nachfrageseite erfüllt sein. Erstens müssen die Konsumenten gut über die Kosten und die Eigenschaften von Gütern und Dienstleistungen informiert sein. Diese Informationen müssen zum einen verfügbar und mit vertretbarem Aufwand zugänglich sein - zum anderen müssen die Verbraucher aber auch in der Lage sein, den Gehalt der zugänglichen Informationen verstehen und verarbeiten zu können. Zweitens müssen Verbraucher in der Lage sein, die Konsequenzen ihrer Wahlentscheidungen abzuschätzen und im Vergleich zu Alternativen abzuwägen. Dies ist insbesondere dann relevant, wenn Entscheidungen nur sehr selten getroffen werden und deswegen die Sammlung von Erfahrungswissen nur sehr eingeschränkt möglich ist. Drittens müssen Verbraucher rational entscheiden. Das bedeutet im Wesentlichen, dass die Betroffenen ihre Entscheidungen auf der Grundlage ihrer jeweiligen Präferenzen treffen und sich durch die Entscheidung nicht selbst schaden. Zur Rationalität des Entscheidungsverhaltens gehört aber auch, dass Entscheidungen vorausschauend getroffen werden (Rice und Unruh 2016).

\subsection{Wahlentscheidungen und pflegerische Versorgung}

Individuelle Wahlentscheidungen spielen in der pflegerischen Versorgung eine herausragende Rolle. In diesem Zusammenhang fällt auf, dass der Gesetzgeber den Versicherungsmarkt und den Leistungsmarkt in der gesundheitlichen und in der pflegerischen Versorgung in sehr unterschiedlicher Art und Weise reguliert hat.

In der Pflegeversicherung spielen Wahlentscheidungen auf dem Versicherungsmarkt allenfalls indirekt eine Rolle. Die wettbewerbliche Orientierung des Versicherungsmarkts ist gering, weil die Versicherten keine eigenständige Wahlentscheidung für eine Pflegekasse treffen bzw. getroffen haben. In der Krankenversicherung haben Wahlentscheidungen dagegen im Grundsatz eine große Bedeutung, weil die Versicherten zwischen unterschiedlichen gesetzlichen Krankenversicherungen entsprechend ihren individuellen Vorlieben wählen können. Dieser Wahlmechanismus ist in der Pflegeversicherung außer Kraft gesetzt, weil die Ver- 
sicherten immer in der gleichen Kranken- und Pflegekasse versichert sind.

Ganz anders ist die Situation auf dem Leistungsmarkt. Im Rahmen der Krankenversicherung haben die Versicherten zwar im Grundsatz auch die freie Wahl zwischen unterschiedlichen Leistungsanbietern wie Ärzten und Krankenhäusern. Von einem Markt kann aber nur eingeschränkt die Rede sein, weil die finanziellen Konsequenzen der Wahlentscheidungen der Versicherten hier ausgesprochen gering sind. Dies ist die Konsequenz dessen, dass die gesetzliche Krankenversicherung sich an der Deckung des Bedarfs gesundheitlicher Versorgung der gesetzlich Versicherten orientiert. Im Gegensatz dazu finanziert die gesetzliche Pflegeversicherung nur einen Teil des Bedarfs an Pflegeleistungen der Versicherten.

Diese subsidiäre Ausrichtung der Pflegeversicherung hat zur Konsequenz, dass Entscheidungen der Versicherten auf dem Leistungsmarkt weitreichende finanzielle Konsequenzen für die Betroffenen selbst haben. Diese Entscheidungen finden auf drei Ebenen statt. Erstens müssen sich Pflegebedürftige in der ambulanten pflegerischen Versorgung zwischen Pflegesachleistung und Pflegegeld bzw. einer Kombinationsleistung entscheiden. Zweitens müssen Pflegebedürftige am Übergang zwischen ambulanter und stationärer pflegerischer Versorgung in ihre Entscheidung über einen Verbleib in der eigenen Häuslichkeit oder einen Umzug in ein Pflegeheim mit einbeziehen, dass bei einer stationären Versorgung ein Eigenanteil zu leisten ist. Ist die Entscheidung für eine stationäre Versorgung gefallen, dürfte drittens die Entscheidung über Ort und Art der Unterbringung nicht unwesentlich von der Höhe des Eigenanteils abhängig sein.

Aus ökonomischer Sicht sind damit ein Teil der Voraussetzungen für die effektive Steuerung des Leistungsmarkts auf der Grundlage von Wahlentscheidungen der Betroffenen im Grundsatz vorhanden. Individuelle Abwägungen zwischen Kosten und Nutzen der zur Verfügung stehenden Alternativen würden damit auch zur Qualitätssicherung beitragen. Leistungsanbieter mit einer unattraktiven Relation von Preis und Qualität würden auf einem funktionierenden Leistungsmarkt nicht nachgefragt und müssten entweder ihr Angebot verbessern oder aus dem Markt ausscheiden.

\subsection{Wahlentscheidungen auf dem Leistungsmarkt}

Trotz der aus ökonomischer Sicht zumindest in Teilen vorliegenden Voraussetzungen für eine effektive Steuerung des Leistungsmarkts durch den Verbraucher sind dessen Wahlentscheidungen nur sehr eingeschränkt in der Lage, die Qualität der pflegerischen Versorgung zu sichern. Die Ursachen hierfür liegen erstens in einer sehr eingeschränkten Rationalität der Wahlentscheidungen, zweitens in einer massiven Einschränkung der Konsumentensouveränität und drittens in der sehr begrenzten Verfügbarkeit von Informationen.

\section{- Eingeschränkte Rationalität der Wahlentscheidungen}

Rational wäre aus Sicht des Verbrauchers - mithin des Pflegebedürftigen -, die Entscheidung vor Eintritt der Pflegebedürftigkeit langfristig vorzubereiten. Diese Entscheidungsvorbereitung müsste darin bestehen, dass die Verbraucher zeitlich deutlich vor Eintritt der Pflegebedürftigkeit Informationen über potenzielle Pflegearrangements, deren Vor- und Nachteile sowie Preise einholen. Nach aktuellen Befragungsdaten suchen immerhin $45 \%$ der befragten Nutzerinnen und Nutzer der Onlinesuchportale der »Weissen Liste « zu Pflegeanbietern vorausschauend und ohne Handlungsdruck nach Informationen über Pflegeanbieter. $31 \%$ der Befragten waren wegen einer akuten Pflege- und Notsituation auf der Suche nach Informationen (Strotbek et al. 2017, S. 28). Es ist allerdings offen, ob die befragte Stichprobe tatsächlich repräsentativ für die Grundgesamtheit der Betroffenen ist. Es ist vielmehr davon auszugehen, dass es eher als unangenehm empfunden wird, sich mit verschiedenen Pflegearrangements - womöglich noch außerhalb der eigenen Häuslichkeit - zu befassen und dies daher verdrängt bzw. aufgeschoben wird. Daher überrascht es auch nicht, dass in einer anderen Befragung der Anteil derjenigen, die schon selbst Maßnahmen für die eigene pflegerische Versorgung getroffen haben, eher niedrig ist (Zok 2015).

Die grundsätzlichen und häufig mit langfristigen Konsequenzen verbundenen Entscheidungen auf dem Leistungsmarkt - ob in der häuslichen oder in der stationären Langzeitpflege - geschehen daher 
häufig kurzfristig unter Zeitdruck. Gerade beim Übergang aus der akuten stationären oder rehabilitativen Versorgung müssen Entscheidungen häufig dringlich getroffen werden - zumal dann, wenn das Angebot freier stationärer Versorgungseinrichtungen begrenzt und eine ambulante Versorgung nicht oder nicht mehr möglich ist.

Außerdem ist die Entscheidungsfähigkeit der Betroffenen in dieser Situation häufig bereits stark eingeschränkt. Dies ist vor allem der Fall, wenn die Pflegebedürftigkeit aufgrund kognitiver Einschränkungen eintritt - schließlich handelt es sich um eine Bevölkerungsgruppe, die sowohl gesundheitlich als auch sozial vulnerabel ist (Ewers et al. 2017, S. 243). Zudem spielt bei der Entscheidung über eine Pflegeeinrichtung häufig die regionale Erreichbarkeit besonders für die Angehörigen - eine größere Rolle als öffentlich zugängliche Qualitätsindikatoren (Kumpunen et al. 2014).

\section{- Einschränkung der Konsumenten- souveränität bei der Wahlentscheidung}

Die Effektivität von Wahlentscheidungen auf dem Leistungsmarkt wird - ökonomisch formuliert auch durch eine massive Einschränkung der Konsumentensouveränität beeinträchtigt. Gerade bei der Wahl unterschiedlicher Pflegearrangements ist die Souveränität der eigenen Entscheidungen sehr begrenzt. Aus einer gesundheitswissenschaftlichen Perspektive besteht ein »Ungleichgewicht zwischen Ressourcen und Anforderungen " (Ewers et al. 2017, S. 237). Die oben angesprochene Dringlichkeit der Entscheidungen in einer belastenden Entscheidungssituation und die Einschränkungen der eigenen Gesundheitskompetenz führen dazu, dass Pflegebedürftige im Regelfall bei der Entscheidung auf die Unterstützung durch Angehörige, Freunde oder institutionelle organisierte Pflegeberatung angewiesen sind.

Gerade Angehörige haben aber nicht zwingend die gleichen Präferenzen und Interessen wie die Pflegebedürftigen selbst. Angehörige haben neben dem Interesse an einer qualitativ hochwertigen pflegerischen Versorgung auch eigene finanzielle Interessen. Diese können darin bestehen, das Vermögen des Pflegebedürftigen - und damit potenziell das eigene Erbe bzw. das eigene Vermögen vor dem Zugriff der Sozialhilfeträger zu schützen.
Auf den ersten Blick widersprechen aktuelle Befunde zum Informationsbedürfnis von Pflegebedürftigen und deren Angehörigen dieser Analyse. Danach identifizieren $36 \%$ der Pflegebedürftigen und 34,1 \% der Angehörigen Kosten als entscheidenden »Qualitätsbereich« (vgl. Abbildung 9.2 im Beitrag von Sonntag et al., $>$ Kap. 9 in diesem Band). Von divergierenden Interessen zwischen Pflegebedürftigen und Angehörigen kann auf den ersten Blick demzufolge nicht gesprochen werden. Aus den Befragungsdaten geht aber nicht hervor, welche Kriterien bei der Auswahl von Pflegeeinrichtungen letztlich entscheidend sind und inwieweit Pflegebedürftige die Interessen ihrer Angehörigen bei der Auswahl internalisieren.

Dieser Interessengegensatz ist noch deutlicher zwischen Pflegebedürftigen auf der einen Seite und Sozialhilfeträgern auf der anderen Seite zu konstatieren. Pflegebedürftige mit einem Anspruch auf Hilfe zur Pflege haben ein Interesse an einer möglichst qualitativ hochwertigen Einrichtung, während der Sozialhilfeträger vor allem ein Interesse an der Begrenzung der Eigenanteile hat. In der Summe begünstigt die Einschränkung der Konsumentensouveränität durch Dritte - seien es Angehörige oder Sozialhilfeträger - den Preiswettbewerb zu Lasten des Qualitätswettbewerbs auf dem Leistungsmarkt.

\section{- Begrenzte Verfügbarkeit von Informationen für die Wahlentscheidung}

Letztlich werden die Wahlentscheidungen durch die mangelnde Verfügbarkeit aussagefähiger Informationen über die Qualität unterschiedlicher Leistungsanbieter massiv beeinträchtigt. Die vorliegenden Informationen sind für die Betroffenen schwer $\mathrm{zu}$ überschauen, wenig aussagekräftig und wenig nutzerfreundlich aufbereitet (Ewers et al. 2017). ${ }^{2}$ Es ist daher wenig überraschend, dass nach einer kürzlich veröffentlichten Erhebung zur Gesundheitskompetenz in Deutschland 58,6\% der Befragten Informationsdefizite über die Qualität von Alten-

2 Zu der Veröffentlichung der Ergebnisse der Qualitätsprüfungen im Rahmen der Pflege-Transparenzvereinbarungen (PTV) und der Vergabe einer Schulnote für die Qualität der Pflegeleistungen vgl. ausführlich den Beitrag von Büscher et al. in diesem Band ( $\triangleright$ Abschn. 4.3). 
und Pflegeheimen identifiziert haben. Etwas niedriger lag der Wert mit 55,5\% der Befragten bei Informationsdefiziten über die Qualität von ambulanten Pflegediensten. Diese Werte sind insofern auffällig, als nur die Informationsdefizite über Anlaufstellen bei Verdacht auf Behandlungsfehler $(61,0 \%)$ noch etwas höher eingeschätzt wurden. Die Informationsdefizite über die Qualität von Krankenhäusern (38,9 \%) und von Ärzten (34,2 \%) wurden als deutlich geringer empfunden (Schaeffer et al. 2016, S. 70).

Vor diesem Hintergrund müssen auch Beratungsangebote - etwa durch die Pflegestützpunkte oder wie im Pflegestärkungsgesetz III vorgesehen durch die Kommunen - ins Leere laufen (Greß 2016). Zudem scheuen Pflegebedürftige vor allem wegen sozialer oder psychischer Barrieren ohnehin den Weg in Beratungsstellen (Ewers et al. 2017). Letztlich verbleibt für den Pflegebedürftigen als aussagefähiger Vergleichsparameter vor allem der Preis (Geraedts et al. 2011).

\subsection{Lösungsansätze}

Vor dem Hintergrund der oben beschriebenen Beeinträchtigungen der Wahlentscheidungen auf dem Leistungsmarkt - vor allem in Hinblick auf eine Begünstigung des Preiswettbewerbs zu Lasten des Qualitätswettbewerbs - stellt sich die Frage, inwieweit die Rahmenbedingungen für diese Wahlentscheidungen verbessert bzw. durch Steuerungsansätze durch den Staat bzw. Verbände ergänzt werden können.

Zur Optimierung der Wahlentscheidungen werden insbesondere Ansätze diskutiert, tatsächlich aussagefähige Informationen über relevante Qualitätsparamater bzw. Qualitätsunterschiede zu veröffentlichen. Dabei müsste das Informationsinteresse von Pflegebedürftigen im Vordergrund stehen und als Grundlage für die rechtzeitige Vorbereitung von Entscheidungen dienen können (Strotbek et al. 2017).

Die Veröffentlichung aussagefähiger Informationen über relevante Qualitätsparamater ist für die Optimierung von Wahlentscheidungen auf dem Leistungsmarkt notwendig - aus Sicht des Autors aber nicht hinreichend. Die Einschränkung der
Konsumentensouveränität durch Dritte bliebe bestehen, weil deren Interesse an der Wahlentscheidung der Pflegebedürftigen nicht neutralisiert wird. Das wird auch so bleiben, solange durch die Pflegeversicherung nur ein Teil des Bedarfs finanziert wird und das Budgetprinzip das Bedarfsprinzip dominiert. Als Konsequenz ist festzuhalten, dass die Funktionsfähigkeit der Qualitätssicherung für die pflegerische Versorgung über Wahlentscheidungen der Betroffenen auch dann weiterhin eingeschränkt bleiben wird, wenn aussagefähige, verständliche und vergleichbare Informationen vorliegen.

Demzufolge müssten staatliche und verbandliche Steuerungsmechanismen gezielt eingesetzt werden, um die Qualität in der Langzeitpflege sichern zu können. Die bisherigen Erfahrungen sind jedoch nicht in allen Bereichen positiv. Beispielhaft lässt sich dies an dem Prozess um die Implementation der in Überarbeitung befindlichen Pflegenoten zeigen. Die geringe Aussagekraft dieser Informationen für die Nutzer lässt sich nicht zuletzt darauf zurückführen, dass das letztlich implementierte System Ergebnis eines Aushandlungsprozesses war und sich im Ergebnis nicht an den sachlichen Notwendigkeiten, sondern an der Zustimmung der beteiligten Akteure orientierte.

Auch in anderen Bereichen führt ein Mix aus staatlicher und verbandlicher Steuerung nicht unbedingt $\mathrm{zu}$ einer sinnvollen Kompensation der Defizite des Marktes bei der Sicherung von Qualität in der pflegerischen Versorgung. Dies lässt sich im Hinblick auf die Personalausstattung in der stationären Pflege illustrieren. Es ist empirisch gut belegt, dass der Strukturparameter Personalausstattung ein wichtiger Bestimmungsfaktor für den Ergebnisparameter Pflegequalität ist (vgl. den Beitrag von Greß und Stegmüller, Kap. 14 in diesem Band). Pflegebedürftige können aus den veröffentlichten Informationen jedoch nicht erkennen, wie sich dieser Strukturparameter zwischen unterschiedlichen Einrichtungen unterscheidet. Dazu müssten Informationen über die Betreuungsrelation von Pflegebedürftigen zu Pflegekräften und die Fachkraftquote in den einzelnen Einrichtungen öffentlich verfügbar sein. Dies ist allerdings nicht der Fall. 


\subsection{Diskussion}

Der Gesetzgeber hat im Gegensatz zur gesetzlichen Krankenversicherung in der sozialen Pflegeversicherung dem Wettbewerb auf dem Leistungsmarkt einen hohen Stellenwert eingeräumt. Ein funktionierender Wettbewerb setzt jedoch nicht nur eine hohe Markttransparenz voraus, sondern auch einen souveränen und rational handelnden Verbraucher. Diese Voraussetzungen sind auf dem Leistungsmarkt in der ambulanten wie der stationären Pflege bisher nur sehr eingeschränkt erfüllt. Pflegebedürftige bzw. deren Angehörige können ihre Wahlentscheidungen derzeit nur auf der Grundlage unvollständiger Informationen treffen. Ohne aussagefähige Informationen über wichtige Struktur-, Prozess- und Ergebnisparameter läuft eine Qualitätssicherung über individuelle Wahlentscheidungen und damit auch das Steuerungspotenzial des Marktes ins Leere.

Zentrale verbandliche Steuerungsmechanismen haben die Defizite des Steuerungsmechanismus Markt im Hinblick auf die Sicherung der Qualität der pflegerischen Versorgung bisher nur unvollständig kompensieren können. Dies lässt sich nicht nur am Beispiel des für die pflegerische Ergebnisqualität hoch relevanten Strukturparameters Personalausstattung in der stationären pflegerischen Versorgung illustrieren. Auch die Entwicklung von transparenten und relevanten Qualitätsindikatoren ist bisher an den Interessengegensätzen der Vertragspartner gescheitert.

Selbst wenn es gelingen sollte, transparente und aussagefähige Qualitätsindikatoren zu entwickeln und für den Verbraucher verfügbar und damit auch für eine unabhängige Pflegeberatung nutzbar zu machen, bleibt der Wettbewerb auf dem Leistungsmarkt eingeschränkt funktionsfähig. Anders formuliert wäre der Verbraucher im Idealfall deutlich besser informiert und besser beraten - die Wahlentscheidungen auf dem Leistungsmarkt würden dennoch weiter durch Dritte zu Gunsten des Preiswettbewerbs und zu Ungunsten des Qualitätswettbewerbs beeinflusst werden.

Angehörige und Sozialhilfeträger würden erst dann das Interesse an der Wahl eines möglichst preiswerten Anbieters verlieren, wenn die finanziellen Konsequenzen der Wahlentscheidungen für die Pflegebedürftigen nachhaltig reduziert würden. Dies würde allerdings bedeuten, dass sich der Leistungsumfang in der Pflegeversicherung ähnlich wie in der Krankenversicherung am Bedarf und nicht am zur Verfügung stehenden Budget orientieren würde. Mit anderen Worten müsste die Konstruktion der Pflegeversicherung als Teilleistungsversicherung auf den Prüfstand gestellt werden (Lüngen 2012).

Von einer Ausweitung des Leistungsumfangs in der Pflegeversicherung würden allerdings in erster Linie die kommunalen Haushalte durch eine Entlastung der Sozialhilfeträger sowie die Generation der Erben profitieren. Umgekehrt würden die Beitragszahler in der sozialen Pflegeversicherung belastet werden - was zumindest unter den derzeitigen Rahmenbedingungen der Beitragskalkulation vor allem niedrige und mittlere Einkommen und in geringerem Ausmaß die Versicherten mit hohem Einkommen sowie den Personenkreis der Privatversicherten betreffen würde.

Open Access Dieses Kapitel wird unter der Creative Commons Namensnennung 4.0 International Lizenz (http://creativecommons. org/licenses/by/4.0/deed.de) veröffentlicht, welche die Nutzung, Vervielfältigung, Bearbeitung, Verbreitung und Wiedergabe in jeglichem Medium und Format erlaubt, sofern Sie den/die ursprünglichen Autor(en) und die Quelle ordnungsgemäß nennen, einen Link zur Creative Commons Lizenz beifügen und angeben, ob Änderungen vorgenommen wurden.

Die in diesem Kapitel enthaltenen Bilder und sonstiges Drittmaterial unterliegen ebenfalls der genannten Creative Commons Lizenz, sofern sich aus der Abbildungslegende nichts anderes ergibt. Sofern das betreffende Material nicht unter der genannten Creative Commons Lizenz steht und die betreffende Handlung nicht nach gesetzlichen Vorschriften erlaubt ist, ist für die oben aufgeführten Weiterverwendungen des Materials die Einwilligung des jeweiligen Rechteinhabers einzuholen.

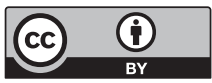




\section{Literatur}

Ewers M, Schaeffer D, Meleis Al (2017) Teach more, do less. Förderung von Health Literacy als Aufgabe der Pflege. In: Schaeffer D, Pelikan JM (Hrsg) Health Literacy. Stand und Perspektiven. Hogrefe, Bern, S 237-257

Geraedts M, Brechtel T, Zöll R, Hermeling P (2011) Beurteilungskriterien für die Auswahl einer Pflegeeinrichtung. In: Böcken J, Braun B, Repschläger U (Hrsg) Gesundheitsmonitor 2011. Bertelsmann-Stiftung, Gütersloh, S 155-172

Greß S (2017) Qualitätssicherung im und durch den Markt für Pflege. In: Spiecker gen. Döhmann I, Wallrabenstein A (Hrsg) Pflegequalität im Institutionenmix. Peter Lang, Frankfurt am Main, S 51-63

Deutscher Bundestag (2016) Schriftliche Stellungnahme zum Entwurf eines Dritten Gesetzes zur Stärkung der pflegerischen Versorgung (PSG III). Deutscher Bundestag, Ausschuss für Gesundheit, Berlin. Ausschussdrucksache 18(14)0204(9)

Kumpunen S, Trigg T, Rodrigues R (2014). Public reporting in health and long-term care to facilitate provider choice. WHO, Copenhagen

Lüngen M (2012) Vollversicherung in der Pflege - Quantifizierung von Handlungsoptionen. Hochschule Osnabrück.

Rice T, Unruh L (2016) The Economics of Health Reconsidered. Fourth Edition. Health Administration Press, Chicago

Schaeffer D, Vogt D, Berens E-M, Hurrelmann K (2016) Gesundheitskompetenz der Bevölkerung in Deutschland. Universität Bielefeld, Fakultät für Gesundheitswissenschaften

Strotbek J et al. (2017) Reformkonzept Verbraucherorientierte Qualitätsberichterstattung in der Pflege. Anforderungen und Lösungsvorschläge mit besonderem Blick auf Lebensqualität, Personalausstattung und Erfahrungswissen. Bertelsmann-Stiftung, Gütersloh

Zok K (2015) Wahrnehmungen zur eigenen Pflegebedürftigkeit. Einstellungen der älteren Bevölkerung zur häuslichen und zur stationären Pflege. WIdOmonitor 12(2):1-15 